\title{
Suicidality and symptoms of anxiety, irritability, and agitation in patients experiencing manic episodes with depressive symptoms: a naturalistic study
}

This article was published in the following Dove Press journal:

Neuropsychiatric Disease and Treatment

31 August 2016

Number of times this article has been viewed

\author{
Jonas Eberhard' \\ Emmanuelle Weiller ${ }^{2}$ \\ 'Department of Clinical Sciences, \\ Lund University, Lund, Sweden; \\ ${ }^{2} \mathrm{H}$. Lundbeck A/S, Copenhagen, \\ Denmark
}

Purpose: Patients with a bipolar I disorder (BD-I) manic episode meeting the Diagnostic and Statistical Manual of Mental Disorders, fifth edition (DSM-5), criteria for "with mixed features" have a high incidence of suicide attempts and of anxiety, irritability, and agitation (AIA) symptoms. The aim of this analysis was to explore the relationship between suicidality and AIA symptoms in patients with BD-I experiencing mania with depressive symptoms, using data from a previous naturalistic study.

Patients and methods: Psychiatrists completed an online questionnaire about their adult patients who had a current BD-I manic episode. Questions covered the DSM-5 "with mixed features" specifier, the severity of AIA symptoms, the frequency and controllability of suicidal ideation, and the number of suicide attempts.

Results: Of 1,035 patients with BD-I mania who were included in the analyses, $348(33.6 \%)$ met the criteria for the DSM-5 "with mixed features" specifier (three or more depressive symptoms). These patients were further stratified according to the severity of their AIA symptoms: "mild AIA" (zero or one AIA symptom above a severity threshold; 105 patients) or "severe AIA" (all three AIA symptoms above a severity threshold; 167 patients). A greater incidence of suicidal ideation was observed in the severe AIA group (71.9\%) than in the mild AIA group (47.6\%). Twice as many patients had easily controlled suicidal ideation than difficult-to-control suicidal ideation in both subgroups. The mean number of suicide attempts was higher in the severe AIA group than in the mild AIA group, during the current episode ( 0.84 vs 0.34 attempts, respectively; $P<0.05$ ) and over the patient's lifetime (1.56 vs 1.04 attempts, respectively).

Conclusion: The high risk of suicide among BD-I mania patients with depressive symptoms is further increased when they experience severe AIA symptoms. Recognizing AIA symptoms in BD-I mania could provide a means of identifying patients with depressive symptoms, as well as those who may be suicidal, thereby allowing for appropriate, tailored treatment.

Keywords: bipolar I disorder, mania with depressive symptoms, suicide, mixed features, DSM-5

\section{Introduction}

Bipolar I disorder (BD-I) is characterized by periods of mania, but patients may also experience periods of depression and "mixed" states in which both manic and depressive symptoms are present. The Diagnostic and Statistical Manual of Mental Disorders, fifth edition (DSM-5), captures mixed states using the "with mixed features" specifier, which is added to a diagnosis of BD-I mania or depression when symptoms of the opposite pole are present. ${ }^{1}$ The DSM-5 lists the following six depressive symptoms in its "with mixed features" specifier for BD-I mania: prominent dysphoria or depressed mood,
Correspondence: Jonas Eberhard H. Lundbeck A/S, Ottiliavej 9, 2500 Valby, Copenhagen, Denmark

Tel +4536432813

Fax +4536301940

Email joeb@lundbeck.com 
diminished interest or pleasure, psychomotor retardation, fatigue or loss of energy, feelings of worthlessness or guilt, and thoughts of death or suicide. ${ }^{1}$ At least three of the depressive symptoms must be present during a manic or hypomanic episode for the specifier to apply. ${ }^{1}$

Mixed states, in general, represent a more severe presentation of BD-I than "pure" manic states. Mixed states are associated with a worse clinical course, a higher rate of comorbid conditions, and a higher incidence of suicide attempts. ${ }^{2,3}$ Indeed, a prospective, naturalistic study, in which physicians completed an online survey about their patients with BD-I mania, revealed that patients meeting the DSM-5 criteria for "with mixed features" were more likely to have made at least one suicide attempt (current episode and lifetime) compared with those not meeting the criteria. ${ }^{4}$ The study also identified a high incidence of anxiety, irritability, and agitation (AIA) symptoms in patients with mixed features (99\%, 97\%, and 95\%, respectively) and a greater severity of AIA symptoms in these patients compared with patients without mixed features. ${ }^{4}$ Similarly, in the IMPACT online survey, a high proportion of patients experiencing BD-I mania with three or more self-reported depressive symptoms also reported both "anxiety or worry" and "irritability or agitation" (72.4\%). ${ }^{5}$ Clearly, AIA symptoms are core features of mixed episodes. They were not included in the DSM-5 "with mixed features" specifier, however, since they occur in both mania and depression and are therefore not specific to either pole. ${ }^{6,7}$

Recognizing AIA symptoms in BD-I mania could provide a means of identifying patients with depressive symptoms, as well as those who may be suicidal, thereby allowing for appropriate treatment. The purpose of the aforementioned prospective, naturalistic study was to determine the prevalence of depressive symptoms according to the DSM-5 "with mixed features" specifier in patients with BD-I mania and to explore any association between depressive symptoms and AIA symptoms or suicidality, among other factors. ${ }^{4}$ The current article builds upon this knowledge by reporting the findings of additional analyses conducted on the naturalistic study dataset to explore the relationship between suicidality and AIA symptoms in patients with BD-I experiencing mania with depressive symptoms.

\section{Patients and methods}

The design and methodology of the prospective, naturalistic study from which the present dataset was obtained have been reported earlier. ${ }^{4}$ Briefly, the study was conducted in compliance with relevant codes of conduct from the
European Pharmaceutical Market Research Association and the Association of the British Pharmaceutical Industry. As a market research study, Clinical Research Ethics Committee or Independent Review Board approval was not required. ${ }^{8}$ Patients gave written informed consent to data collection and use, prior to its collection. Patient data were anonymized, stored, and processed in agreement with the requirements of the EU Data Protection Directive 95/46/EC.

The study surveyed representative psychiatrists from centers in Australia, Brazil, Canada, Germany, Italy, Spain, Turkey, and the UK, between October 2013 and March 2014. To be eligible for inclusion, psychiatrists were required to be actively managing and treating adult patients diagnosed with BD-I, to have been qualified for a minimum of 3 years ( 2 years in Australia), and to have a caseload of $\geq 20$ patients with BD-I per month ( $\geq 14$ per month in Australia), including eight or more patients with a BD-I manic episode per month (three or more per month in Australia; five or more per month in Germany and the UK). The patients of these psychiatrists were required to be $\geq 18$ years of age, with a diagnosis of BD-I according to the Diagnostic and Statistical Manual of Mental Disorders, fourth edition, Text Revision (DSMIV-TR), criteria, ${ }^{9}$ and with a current manic episode of onset within the last 3 months.

The psychiatrists completed an online questionnaire, which was translated into their local language, for each of their eligible patients (up to six patients per psychiatrist). Table 1 lists the questions of interest for the current analysis. The questionnaire included six questions to assess the presence of depressive symptoms in BD-I mania, based upon the DSM-5 "with mixed features" specifier. The questionnaire also included a global assessment of the severity of AIA symptoms, with each symptom being rated on a scale from 1 (absent) to 7 (very severe). Suicidal ideation during the current manic episode was assessed in terms of frequency and controllability. The number of suicide attempts was recorded for the current manic episode and for the patient's lifetime. Finally, patient demographics and disease history were recorded. For a full list of assessments in the physician survey, the readers are referred to the primary manuscript. ${ }^{4}$

The same "matched" dataset was used as in the primary, naturalistic study, comprising patients for whom data were available from both the physician assessment and a patientreported Mini International Neuropsychiatric Interview (MINI) module (used in the naturalistic study to assess the presence or absence of depressive features from the 
Table I Questions relating to depressive symptoms, AIA symptoms, and suicidality from an online questionnaire for psychiatrists regarding their patients with $B D-I$

\section{DSM-5 criteria}

As far as you are aware, did the patient experience any of the following symptoms almost every day since the beginning of their most recent (current) manic episode?

- Prominent dysphoria or depressed mood as indicated by either subjective report (eg, feels sad or empty) or observation made by others (eg, appears tearful)

- Diminished interest or pleasure in all, or almost all, activities (as indicated by either subjective account or observation made by others)

- Psychomotor retardation nearly every day (observable by others, not merely subjective feelings of being slowed down)

- Fatigue or loss of energy

- Feelings of worthlessness or excessive or inappropriate guilt (not merely self-reproach or guilt about being sick)

- Recurrent thoughts of death (not just fear of dying), recurrent suicidal ideation without a specific plan, or a suicide attempt or a specific plan for committing suicide

Possible answers for each symptom: yes or no

Global assessment of symptoms

Considering your clinical experience with all patients with BD-I, how severe have the following symptoms been during their most recent (current) manic episode?

- Anxiety

- Irritability

- Agitation

Possible answers for each symptom: I) absent, 2) minimally severe,

3) mildly severe, 4) moderately severe, 5) markedly severe, 6) severe, or

7) very severe

\section{Suicidal ideation}

Based on frequency and controllability, how severe has the suicidal ideation been since the beginning of their most recent (current) manic episode? Suicidal ideation includes thoughts about a wish to be dead and suicidal thoughts about killing oneself with or without intent to act.

Possible answers:

- No suicidal ideation

- Few events (once a week), easily controlled

- Few events (once a week), difficult to control

- Frequent events (2-5 times a week), easily controlled

- Frequent events (2-5 times a week), difficult to control

- Very frequent events (daily), easily controlled

- Very frequent events (daily), difficult to control

Suicide attempts - lifetime

How many suicide attempts has the patient made in their lifetime?

Suicide attempts include any potentially self-injurious act committed with at least some wish to die

Possible answers: 0 times, I time, 2 times, $3-4$ times, $\geq 5$ times, don't know

\section{Suicide attempts - most recent}

How many suicide attempts did the patient make/has the patient made in their most recent (current) manic episode? Suicide attempts include any potentially self-injurious act committed with at least some wish to die. (Question skipped if the previous question was answered "0 times") Possible answers: 0 times, I time, 2 times, $\geq 3$ times

Note: Adapted from Young AH, Eberhard J. Evaluating depressive symptoms in mania: a naturalistic study of patients with bipolar disorder. Neuropsychiatr Dis Treat. 2015;11:1137-1143. ${ }^{4}$

Abbreviations: AIA, anxiety, irritability, and agitation; BD-I, bipolar I disorder: DSM-5, Diagnostic and Statistical Manual of Mental Disorders, fifth edition. patient's perspective; not otherwise relevant for the current analysis). ${ }^{4}$ Based upon the physician survey, patients were stratified according to whether or not they met the criteria for the DSM-5 "with mixed features" specifier (three or more depressive symptoms). Only patients with mixed features were included in the suicidality analyses. These patients were further stratified according to the severity of their AIA symptoms. The "severe AIA" group comprised patients with a severity rating of $\geq 4$ points (moderately severe to very severe) for all three AIA symptoms. The "mild AIA" group comprised patients with no AIA symptoms with a severity rating of $\geq 4$ points, or with only one symptom with a severity rating of $\geq 4$ points. The remaining group of patients with two AIA symptoms with a severity rating of $\geq 4$ points was not included in further analyses.

Patient characteristics were analyzed using descriptive statistics. Statistical comparisons in terms of suicidal ideation and suicide attempts between the severe AIA and mild AIA patient subgroups were made using Fisher's exact test, for differences in proportions, and a two-sided $t$-test, for differences in means. Values of $P<0.05$ were considered to be statistically significant.

\section{Results \\ Population characteristics}

Data from 1,035 patients with BD-I mania (DSM-IV-TR criteria) were included in the analyses, which were reported by 184 physicians. Patients had a mean age of 40 years (range 18-73 years); $52 \%$ were female; and $44 \%$ were in employment.

Overall, 348 patients (33.6\%) had three or more depressive symptoms during their current manic episode, and thus they met the criteria for the DSM-5 "with mixed features" specifier. Of these patients, 105 (30.2\%) had zero or one AIA symptom with a severity rating of $\geq 4$ points and formed the "mild AIA" group. In contrast, 167 patients (48.0\%) had all three AIA symptoms with a severity rating of $\geq 4$ points, forming the "severe AIA" group. Finally, 76 patients $(21.8 \%)$ had two AIA symptoms with a severity rating of $\geq 4$ points. Figure 1 shows the proportion of patients with each AIA symptom and the overlap between symptoms.

\section{Suicidal ideation, stratified by AIA severity}

Among patients with a BD-I manic episode with mixed features, suicidal ideation had a greater incidence in the severe AIA group (120/167 patients; $71.9 \%)$ than in the mild AIA group (50/105 patients; 47.6\%). Figure 2 shows 


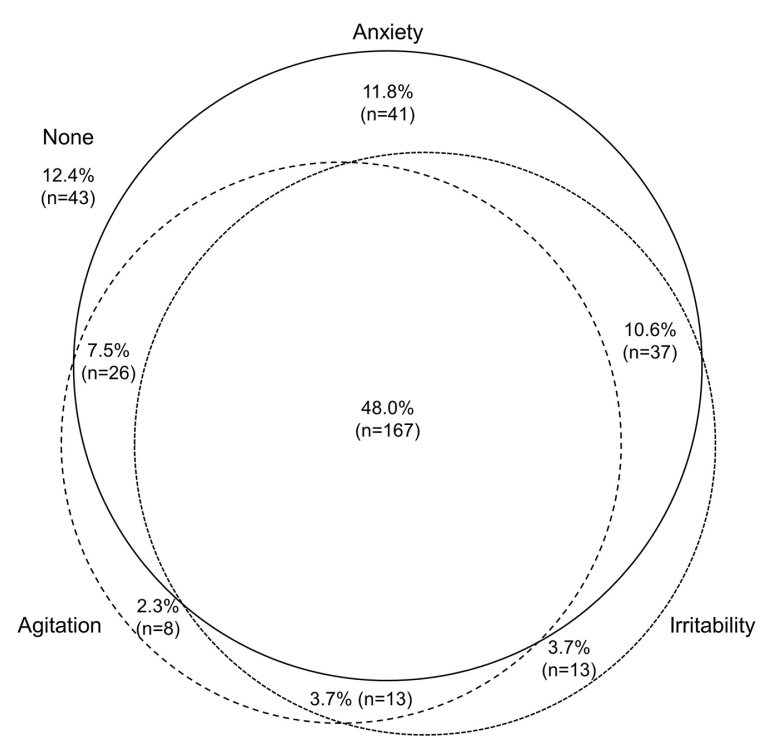

Figure I Venn diagram displaying the overlap of comorbid AIA symptoms in BD-I mania with mixed features.

Notes: The severity of each AIA symptom was rated by psychiatrists from $\mathrm{I}=$ absent to $7=$ very severe, for 348 patients with BD-I mania with mixed features (DSM-5 specifier). A severity rating of $\geq 4$ points (moderately severe to very severe) indicated that a symptom was present.

Abbreviations: AIA, anxiety, irritability, and agitation; BD-I, bipolar I disorder; DSM-5, Diagnostic and Statistical Manual of Mental Disorders, fifth edition.

the frequency and controllability of suicidal ideation, split by the AIA severity group. Suicidal ideation was more frequent in the severe AIA group than the mild AIA group for each frequency category (ie, few events, frequent events, and very frequent events). Eleven patients $(6.6 \%)$ in the severe
AIA group had very frequent suicidal ideation, compared with no patients $(0.0 \%)$ in the mild AIA group. In the severe AIA group, twice as many patients had easily controlled suicidal ideation (81 patients) than difficult-to-control suicidal ideation (39 patients). This ratio was the same in the mild AIA group, where 34 patients had easily controlled suicidal ideation and 16 patients had difficult-to-control suicidal ideation.

\section{Suicide attempts, stratified by AIA severity}

The mean number of suicide attempts during the current episode in patients with BD-I mania with mixed features was statistically significantly higher in the severe AIA group than in the mild AIA group ( 0.84 vs 0.34 suicide attempts, respectively; $P<0.05 ; \mathrm{n}=162$ [question skipped for 110 patients with zero lifetime suicide attempts]). Figure 3 shows the number of suicide attempts in the current manic episode, stratified by AIA severity. The proportion of patients with 1,2 , or $\geq 3$ suicide attempts in the current episode was greater in the severe AIA group than in the mild AIA group. The proportion of patients with no suicide attempts in the current episode was statistically significantly lower in the severe AIA group than in the mild AIA group $(P<0.05)$.

The mean number of lifetime suicide attempts was also higher in the severe AIA group than in the mild AIA group

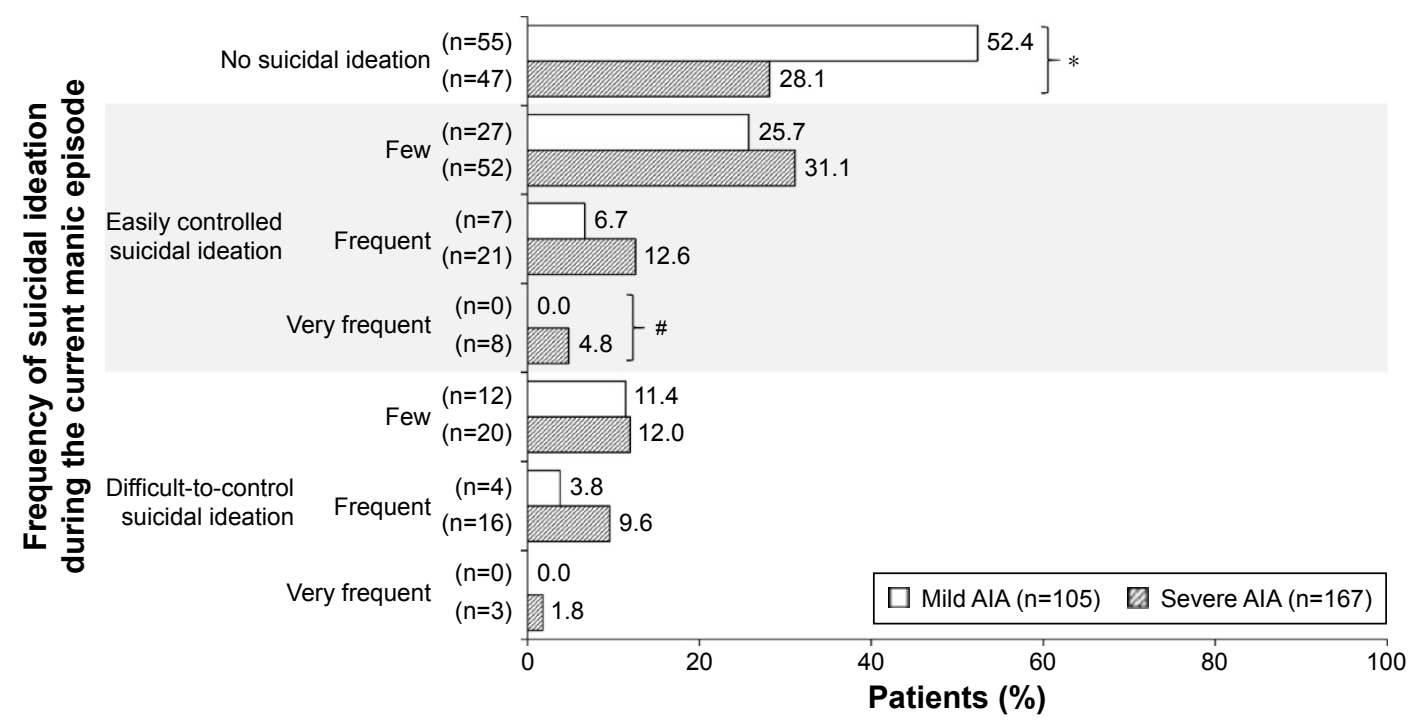

Figure 2 The frequency and controllability of suicidal ideation in the current manic episode, stratified by AIA severity, in BD-I mania with mixed features. Notes: The severity of each AIA symptom was rated by psychiatrists from I= absent to $7=$ very severe, for 348 patients with BD-I mania with mixed features (DSM-5 specifier). The severe AIA group comprised patients with a severity rating of $\geq 4$ points (moderately severe to very severe) for all three AIA symptoms. The mild AIA group comprised patients with a severity rating of $\geq 4$ for zero or one AIA symptom. Frequency of suicidal ideation was categorized as follows: no suicidal ideation, few events (once a week), frequent events ( $2-5$ times a week), or very frequent events (daily), since the beginning of the current manic episode. Controllability of suicidal ideation was categorized as easily controlled or difficult to control. $* P<0.05$ between groups; ${ }^{*} P<0.05$ between groups but the sample size was very small ( $<30$ patients). Abbreviations: AIA, anxiety, irritability, and agitation; BD-I, bipolar I disorder; DSM-5, Diagnostic and Statistical Manual of Mental Disorders, fifth edition. 


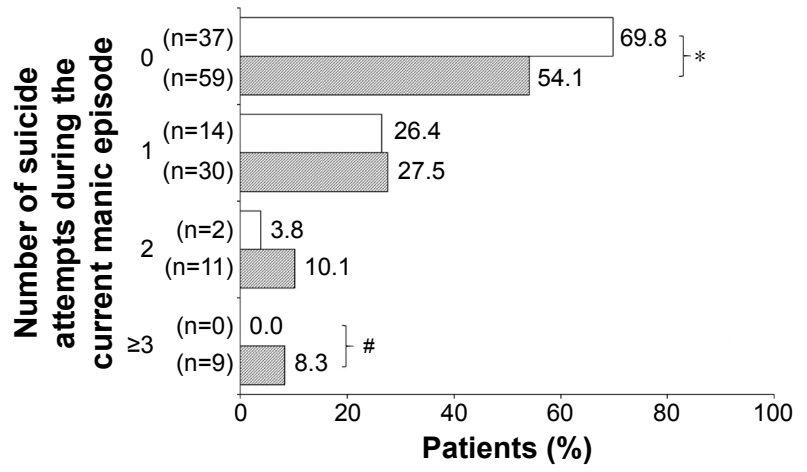

Mild AIA (n=53) Severe AIA (n=109)

Figure 3 The number of suicide attempts in the current manic episode, stratified by AIA severity, in BD-I mania with mixed features.

Notes: The severity of each AIA symptom was rated by psychiatrists from I= absent to $7=$ very severe, for 348 patients with BD-I mania with mixed features (DSM-5 specifier). The severe AIA group comprised patients with a severity rating of $\geq 4$ points (moderately severe to very severe) for all three AIA symptoms. The mild AIA group comprised patients with a severity rating of $\geq 4$ for zero or one AIA symptom. ${ }^{*} P<0.05$ between groups; ${ }^{\sharp}<0.05$ between groups but the sample size was very small ( $<30$ patients)

Abbreviations: AIA, anxiety, irritability, and agitation; BD-I, bipolar I disorder; DSM-5, Diagnostic and Statistical Manual of Mental Disorders, fifth edition.

( 1.56 vs 1.04 suicide attempts, respectively; $\mathrm{n}=226$ [excluding 46 patients whose physician answered “don't know"]). The proportion of patients with $1,3-4$, or $\geq 5$ lifetime suicide attempts was greater in the severe AIA group than in the mild AIA group $(22.7 \%$ vs $13.8 \%, 12.1 \%$ vs $4.3 \%$, and $7.6 \%$ vs $4.3 \%$, respectively; $P<0.05$ between groups for three to four suicide attempts, but the sample size was very small $[<30$ patients]). The proportion of patients with no lifetime suicide attempts, or two suicide attempts, was lower in the severe AIA group than in the mild AIA group (43.9\% vs $55.3 \%$, and $13.6 \%$ vs $22.3 \%$, respectively).

\section{Discussion}

In these analyses of physician survey data from a prospective, naturalistic study, one-third of patients with BD-I (33.6\%) experienced three or more depressive symptoms during their current manic episode, thereby meeting the criteria for the DSM-5 "with mixed features" specifier. This proportion is similar to that in the IMPACT survey, where $39.3 \%$ of patients self-reported three or more depressive symptoms in a previous manic episode. ${ }^{5}$ Furthermore, in post hoc analyses of large datasets from randomized, controlled trials in patients with a BD-I manic or mixed episode (DSM-IV or DSM-IV-TR criteria), the proportion of patients meeting proxy criteria for the DSM-5 "with mixed features" specifier was $33.7 \%$ (aripiprazole trials), $34.2 \%$ (asenapine trials), and $28.0 \%$ (olanzapine trials). ${ }^{10-12}$
DSM-5 mania with depressive symptoms is a severe presentation of BD-I, and the management of such patients is extremely challenging. Mixed states are associated with a greater burden of disease, shorter symptom-free periods, and a greater incidence of treatment dissatisfaction among clinicians, than if depressive symptoms are absent during mania. ${ }^{4,5}$ Mixed states, being difficult to treat, are also associated with longer hospital stays than "pure" manic or depressive episodes. ${ }^{13}$

BD-I is strongly associated with suicide. The absolute risk of suicide in $\mathrm{BD}$ was estimated in a national cohort study to be $7.8 \%$ in men and $4.8 \%$ in women (compared with $0.7 \%$ and $0.3 \%$, respectively, in a nonpsychiatric population).${ }^{14}$ Furthermore, patients with a BD-I mixed state have a clinically relevant higher risk of suicidal ideation than those with a non-mixed state. ${ }^{15,16}$ Consequently, in the current analyses of patients with BD-I mania with mixed features, the risk of suicidal ideation was inherently high. However, by looking at the incidence of AIA symptoms among patients with mixed features, a large subgroup (48.0\%) with all three AIA symptoms was identified, who were at even greater risk. These patients had a higher overall incidence of suicidal ideation in their current episode and were more likely to have multiple events of suicidal ideation per week, compared with patients with zero or one AIA symptom. Furthermore, the presence of all three AIA symptoms in patients with mixed features was associated with a higher number of suicide attempts (lifetime and current episode) and a lower probability of having made no suicide attempts. This aligns with previous research showing that severe anxiety is a clinically relevant risk factor for suicide in BD. ${ }^{15}$

While providing an important "real-world" insight into the clinical relevance of AIA symptoms in BD-I with mixed features, this naturalistic study had a number of limitations. The analysis was based on a market research questionnaire, rather than a clinical rating scale, meaning that the validity and reliability of the questions (in multiple languages) are unknown. Nevertheless, in the primary study, individual depressive symptoms were rated consistently between the physician assessment and the patient-reported MINI module. ${ }^{4}$ Another limitation is that the questionnaire did not record what, if any, treatments the patients were receiving. Consequently, the relationship between AIA symptoms and the side effects of medication, or treatment withdrawal effects, cannot be determined. Finally, by their nature, naturalistic studies are limited by a lack of control.

The risk of suicide in BD-I is greatest during the first year after contact with mental health services, highlighting 
the need for early interventions. ${ }^{14}$ Since patients with mixed states are at increased risk of suicide, early and accurate diagnosis is essential to ensure that the optimal treatment is prescribed. Robust tools such as the MINI module, administered as a patient questionnaire, are now available to assess depressive symptoms (according to the DSM-5 "with mixed features" specifier) in patients with BD-I mania. ${ }^{17}$ Furthermore, it is possible that the combination of AIA symptoms could be used as "gateway symptoms" to alert the psychiatrist to those patients who are likely to be experiencing depressive symptoms during a manic episode and who are at increased risk of suicide. A patient presenting with BD-I and AIA symptoms should be fully assessed for mixed features and then treated in a manner that is tailored to their particular needs. In major depressive disorder, for example, anxious mood, irritability, and inner tension are common "trigger symptoms" that lead to the prescription of antipsychotics by physicians. ${ }^{18}$ Thus, by close monitoring of AIA symptoms, and appropriate treatment, it may be possible to reduce the risk of suicide in the high-risk population of patients with BD-I mania with mixed features.

\section{Conclusion}

Patients with BD-I experiencing a manic episode with depressive symptoms have a high risk of suicide, which is further increased when they experience moderately severe to very severe AIA symptoms. Physicians of patients with BD-I should be alert to the presence of subsyndromal AIA symptoms, in order to identify high-risk patients. Finally, the identification of each patient's specific symptoms should facilitate a more tailored, and therefore potentially more effective, approach to treatment.

\section{Acknowledgments}

This study was funded by H. Lundbeck A/S (Copenhagen, Denmark). Data analysis was performed by Ashfield Insight and Performance (Macclesfield, UK). Writing services were provided by Cambridge Medical Communication Ltd (Cambridge, UK).

\section{Author contributions}

Both authors contributed toward data analysis, drafting and revising the paper and agree to be accountable for all aspects of the work.

\section{Disclosure}

Jonas Eberhard and Emmanuelle Weiller are employees of H. Lundbeck A/S. The authors report no other conflicts of interest in this work.

\section{References}

1. American Psychiatric Association. Diagnostic and Statistical Manual of Mental Disorders. 5th ed. Arlington, VA: American Psychiatric Association; 2013. [DSM-5].

2. Vieta E, Valentí M. Mixed states in DSM-5: implications for clinical care, education, and research. $J$ Affect Disord. 2013;148(1):28-36.

3. Swann AC, Lafer B, Perugi G, et al. Bipolar mixed states: an international society for bipolar disorders task force report of symptom structure, course of illness, and diagnosis. Am J Psychiatry. 2013;170(1): $31-42$.

4. Young AH, Eberhard J. Evaluating depressive symptoms in mania: a naturalistic study of patients with bipolar disorder. Neuropsychiatr Dis Treat. 2015;11:1137-1143.

5. Vieta E, Grunze H, Azorin JM, Fagiolini A. Phenomenology of manic episodes according to the presence or absence of depressive features as defined in DSM-5: results from the IMPACT self-reported online survey. J Affect Disord. 2014;156:206-213.

6. Cassidy F. Anxiety as a symptom of mixed mania: implications for DSM-5. Bipolar Disord. 2010;12(4):437-439.

7. Koukopoulos A, Sani G, Ghaemi SN. Mixed features of depression: why DSM-5 is wrong (and so was DSM-IV). Br J Psychiatry. 2013;203(1): $3-5$.

8. European Pharmaceutical Market Research Association (EphMRA) [webpage on the Internet]. Code of Conduct 2016. Available from: http://www.ephmra.org/user_uploads/2016\%20code $\% 20$ of $\% 20$ conduct\%20february\%202016.pdf. Accessed May 26, 2016.

9. American Psychiatric Association. Diagnostic and Statistical Manual of Mental Disorders. 4th ed. Text Revision. Washington, DC: American Psychiatric Association; 2000. [DSM-IV-TR].

10. McIntyre RS, Miguelez M, Vogel Marler S, Baker RA, Rahman Z [webpage on the Internet]. Analysis of aripiprazole clinical data using the proposed DSM-5 diagnostic criteria for mixed features in bipolar disorder. Poster abstract NR8-08 in Abstract Book for American Psychiatric Association 2013 Annual Meeting; May 18-22, 2013; San Francisco, CA. Available from: http://www.psychiatry.org/psychiatrists/search-directories-databases/library-and-archive. Accessed February 9, 2016.

11. McIntyre RS, Tohen M, Berk M, Zhao J, Weiller E. DSM-5 mixed specifier for manic episodes: evaluating the effect of depressive features on severity and treatment outcome using asenapine clinical trial data. J Affect Disord. 2013;150(2):378-383.

12. Tohen M, McIntyre RS, Kanba S, Fujikoshi S, Katagiri H. Efficacy of olanzapine in the treatment of bipolar mania with mixed features defined by DSM-5. J Affect Disord. 2014;168:136-141.

13. Ösby U, Tiainen A, Backlund L, et al. Psychiatric admissions and hospitalization costs in bipolar disorder in Sweden. J Affect Disord. 2009; 115(3):315-322.

14. Nordentoft M, Mortensen PB, Pedersen CB. Absolute risk of suicide after first hospital contact in mental disorder. Arch Gen Psychiatry. 2011; 68(10):1058-1064.

15. Pompili M, Gonda X, Serafini G, et al. Epidemiology of suicide in bipolar disorders: a systematic review of the literature. Bipolar Disord. 2013;15(5):457-490.

16. Valentí M, Pacchiarotti I, Rosa AR, et al. Bipolar mixed episodes and antidepressants: a cohort study of bipolar I disorder patients. Bipolar Disord. 2011;13(2):145-154.

17. Hergueta T, Weiller E. Evaluating depressive symptoms in hypomanic and manic episodes using a structured diagnostic tool: validation of a new Mini International Neuropsychiatric Interview (M.I.N.I.) module for the DSM-5 'with mixed features' specifier. Int J Bipolar Disord. 2013;1:21.

18. McIntyre RS, Weiller E. Real-world determinants of adjunctive antipsychotic prescribing for patients with major depressive disorder and inadequate response to antidepressants: a case review study. Adv Ther. 2015;32(5):429-444. 
Neuropsychiatric Disease and Treatment

Dovepress

\section{Publish your work in this journal}

Neuropsychiatric Disease and Treatment is an international, peerreviewed journal of clinical therapeutics and pharmacology focusing on concise rapid reporting of clinical or pre-clinical studies on a range of neuropsychiatric and neurological disorders. This journal is indexed on PubMed Central, the 'PsycINFO' database and CAS,

and is the official journal of The International Neuropsychiatric Association (INA). The manuscript management system is completely online and includes a very quick and fair peer-review system, which is all easy to use. Visit http://www.dovepress.com/testimonials.php to read real quotes from published authors.

Submit your manuscript here: http://www.dovepress.com/neuropsychiatric-disease-and-treatment-journal 